\title{
Theoretical Study and Application of Rate Transient Analysis on Complex Fractured-Caved Carbonate Reservoirs
}

\author{
Qingyan Yu $\mathbb{D}^{1},{ }^{1}$ Qi Wang $\mathbb{D}^{2}$, Pengcheng Liu $\mathbb{D}^{3},{ }^{3}$ Jing Zhang, ${ }^{2}$ Qi Zhang, ${ }^{2}$ Xiaojuan Deng, ${ }^{2}$ \\ and Kai Feng ${ }^{4}$
}

${ }^{1}$ Research Institute of Petroleum Exploration and Development of SINOPEC, Beijing 100083, China

${ }^{2}$ PetroChina Research Institute of Petroleum Exploration \& Development, Beijing 100083, China

${ }^{3}$ School of Energy Resources, China University of Geosciences (Beijing), Beijing 100083, China

${ }^{4}$ Tarim Oil Field Company, PetroChina, Korla 841000, China

Correspondence should be addressed to Qingyan Yu; yuqingyan.syky@sinopec.com

and Qi Wang; wangqi.riped@petrochina.com.cn

Received 3 November 2020; Revised 26 December 2020; Accepted 13 January 2021; Published 25 January 2021

Academic Editor: Shun Liu

Copyright (C) 2021 Qingyan Yu et al. This is an open access article distributed under the Creative Commons Attribution License, which permits unrestricted use, distribution, and reproduction in any medium, provided the original work is properly cited.

\begin{abstract}
Carbonate reservoirs are mainly fractured-caved reservoirs with very well-developed dissolved pores, fractures, and caves. They have strong heterogeneity with various types of reservoir pore spaces. Using seismic inversion and reservoir static characterization, the result shows that the fractured-caved carbonate rocks in China are mainly caves with poor connectivity and complex oil-water distribution. Large-scale dissolved caves are mostly discrete and isolated, while the fractures are complex and various. The fracture features are observed either as a single large fractures or as a local fracture network. The characteristics of fluid flow in fracture-caved reservoirs vary as a result of the different combinations of fractures and caves. Currently, the static characterization technology of fractured-caved reservoirs is influenced by the limited resolution of seismic data, leading to large interpretation errors. In contrast, the dynamic method is a more reliable and effective method to determine reservoir parameters. However, traditional seepage equations cannot accurately characterize the flow pattern of fractured-caved carbonate reservoirs. In the case of a single large-scale dissolved fractured-caved reservoir, oil wells are usually connected to large caves through large fractures or directly drilled into large dissolved caves. In this study, the large-scale dissolved caved reservoir is simplified into two cases: (1) a single-cave and single-fracture series model composed of a single-cave and a single-fracture and (2) a composite model of dissolved caves and surrounding fracture networks. Note that the flow in a large cave is considered as free flow due to its large scale. The flow in a large fracture connected to the cave is considered as flow through porous media, and the flow in the reservoir surrounding the fracture network is considered as multiple-porosity model seepage flow. The corresponding seepage-free flow coupling mathematical model of different fractured-caved reservoirs has been established on this basis. We also obtained the rate transient analysis type curves of the oil well, conducted sensitivity analysis of each parameter, constructed the corresponding rate transient analysis curves, analyzed sensitivities of each parameter, and finally designed a dynamic evaluation method of well and reservoir parameters for different types of fractured-caved carbonate reservoirs. This study extensively applies this method in the Halahatang Oilfield of China and evaluates parameters such as reservoir reserves and physical properties to provide rational guidance for developing fractured-caved carbonate reservoirs.
\end{abstract}

\section{Introduction}

The fractured-caved reservoir is a vital type of carbonate reservoirs, characterized by strong heterogeneity, various types, complex connectivity, complex fluid flow mechanisms, and complicated gas-water contacts [1-3]. The reservoir is dom- inated by fractures, dissolved caves, and dissolved pores. Most of the dissolved caves are discretely distributed and isolated, and the fractures are highly heterogeneous, either with a single large fracture or a local fracture network [4]. Due to their very low porosity and permeability, matrix pores have minimal seepage and storage capacity. Therefore, they have 


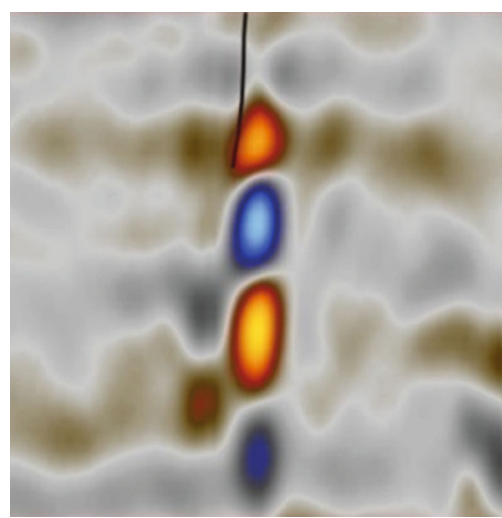

(a)

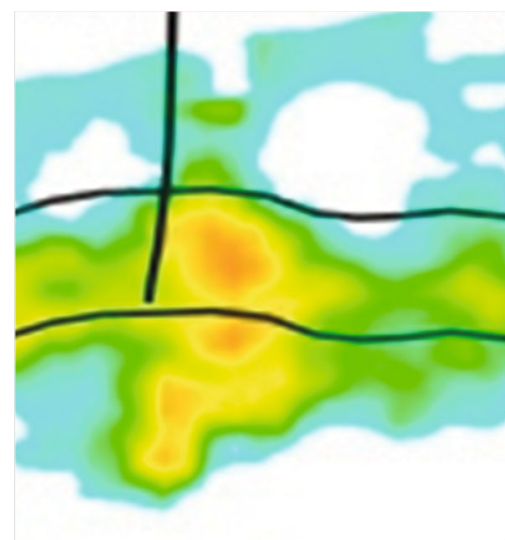

(b)

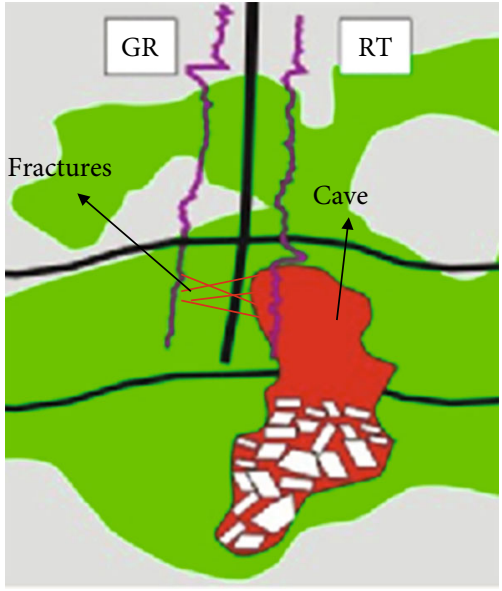

(c)

FIGURE 1: Seismic characteristics, inversion results, and development models of fractured-caved carbonate reservoirs: (a) seismic reflection characteristics of fractured-caved reservoirs; (b) seismic inversion results of fracture-cavity reservoirs; (c) fractured-caved reservoir development schematic diagram.

little influence on the fluid flow of the entire reservoir. Generally, the influence of the matrix does not need to be taken into consideration in the fluid flow of fractured-caved reservoirs [5]. Fractures have significant permeability and are the main seepage channels. Nevertheless, their storage capacity is lower than that of large dissolved caves, so the fracture is generally considered to be a unit with low storage and high seepage and is the primary fluid flow channel [6-10]. Large-scale dissolved caves are units with high storage capacity and high permeability. However, due to the large scale of the caves, their fluid flow is considered to be free flow and no longer Darcy seepage flow [11-13]. The variable scale of fracturedcaved reservoir pores determines the variable rates of fluid flow in the reservoir. The fluid flow of the reservoir presents different characteristics due to the different combinations of fractures and caves [14-16].

For this type of fractured-caved reservoir, the mathematical characterization of continuum models such as dualporosity model, triple-porosity model, multiple-porosity model, and their composite models proposed by previous researchers will no longer be applicable. The characterization method based on the discrete medium model can describe the details of the flow in different fractured caves, but this comes with modeling challenges and large computation limits when applied to well-testing and dynamic analysis theory [17, 18]. Other studies have used the pipe flow and percolationpipe flow coupling model $[19,20]$ and conducted their research based on alternative theories for large fracturedcaved reservoirs, but these studies were not comprehensive. Other researchers have conducted only qualitative research. They have not conducted quantitative research on reservoir parameters and oil well parameters of large fractured-caved reservoirs. As a result, there is no systematic dynamic evaluation method for reservoir parameter evaluation for the fractured-caved reservoirs $[17,18,21]$. Therefore, this study establishes a simplified model which mainly considers large dissolved caves, by simplifying an extensive dissolved caved reservoir. Specifically, there are two large models: a single cave connected to a large fracture and a composite model consisting of a single cave and surrounding reservoirs. A mathematical model of fluid flow, considering seepage-free flow couplings under different combinations, is established. The model is finally adjusted to obtain the corresponding typical curve after rate transient analysis of the oil well. Using this process and the fractured-caved parameter matching method, we can evaluate the dynamic parameter of production wells. What is more, the connectivity of fracture-caved reservoirs can be properly understood through dynamic inversion of fractured-caved reservoirs with large-scale dissolved caves and by obtaining the necessary parameters. These results are of great significance for the proper understanding and development of such fractured-caved reservoirs.

\section{Simplified Discrete Mathematical Model and Rate Transient Analysis Type Curves}

There are various types of connections between fractures and caves within fractured-caved carbonate reservoirs. Also, the relationship between dissolved caves and fractures is complex. However, most fractured-caved carbonate reservoirs are characterized by "bead-shaped" seismic reflection, generally indicating large caves (Figure 1). The existence of these "bead-shaped" fractured-caved reservoirs suggests that this is one of the most common forms of connectivity $[19,20]$. In cases where large-scale dissolved caves have been developed in carbonate reservoirs, the caves are connected to the wellbore through large fractures, or vertical wells are drilled directly on large caves; the capacity and permeability of the matrix cannot be taken into consideration in the reservoir and the reservoir can be described mathematically utilizing a simplified discrete model with seepage-free flow coupling. The following assumptions can be made for the simplified discrete large-scale fracture-cave model: (1) oil wells produce at a stable rate; (2) oil reservoir is a depletion drive reservoir; (3) the fluid is single phase and weakly compressible, and its compression coefficient and volume coefficient are constant; 


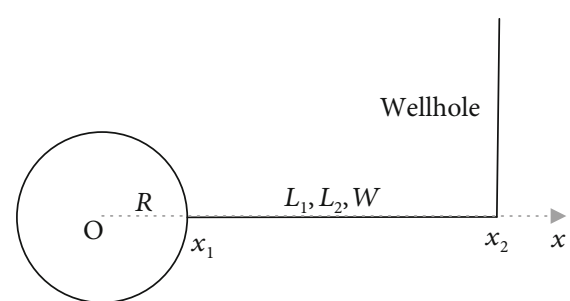

FIGURE 2: Schematic diagram of single-cave and single-fracture series physical model.

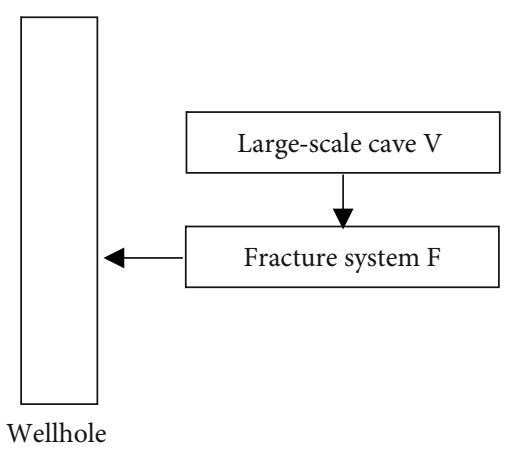

Figure 3: Schematic diagram of single-cave and single-fracture series seepage flow model.

(4) large caves are weakly compressible, and their compressibility is constant; (5) large caves are unfilled or semifilled, as spheres or cylinders with radius $R$, and flow is considered as free flow; (6) the large fracture is flat and the fluid flow in the fracture follows Darcy's law, and the fluid flow in the surrounding multimedium reservoir also follows Darcy's law; and (7) gravity acceleration, wellbore storage effect, and skin effect are ignored.

2.1. Single-Cave and Single-Fracture Series Model. When oil wells are drilled through a large fracture, and the fracture is connected to a large-scale cave (i.e., the large fracture and the cave form a single-cave and single-fracture series model), fluid production ultimately depends on the elastic energy of the large cave, the fracture, and the fluid. Large caves are the main reservoir space, and fractures are the effective secondary reservoir space and the main seepage channel. Large caves cannot supply fluid directly to the wellbore but can indirectly supply fluid through fractures.

2.1.1. Physical Model. Figure 2 shows the single-cave and single-fracture series physical model. Where the cave's central point is the origin $\mathrm{O}$, the intersection point of the cave, and the fracture is $x_{1}$, and the connection point of the fracture and the wellbore is $x_{2}$. It can be seen that the cave radius is $R=x_{1}$, the fracture length is $L_{1}=x_{2}-x_{1}$, the fracture height is $L_{2}$, the fracture length is $W$, and the wellbore radius is $r_{\mathrm{w}}$. The oil well is directly connected to the fractures, and the dissolved caves produce fluid through the fractures. Figure 3 is a schematic diagram of the seepage flow of a single-cave and single-fracture series connection model.
2.1.2. Establishment and Solution of the Mathematical Model. Large caves are the main reservoir spaces, and fractures are the effective reservoir and seepage spaces. The fluid flow capacity in a large cave is robust, and it is considered as free flow, i.e., the fluid flowing in caves results in very little pressure drop. Then, the dimensionless partial differential equation describing fluid flow in the reservoir is:

$$
\begin{aligned}
\frac{\partial^{2} p_{\mathrm{FD}}}{\partial x_{\mathrm{D}}^{2}} & =\omega_{\mathrm{F}} \frac{\partial p_{\mathrm{FD}}}{\partial t_{\mathrm{D}}}, \quad\left(x_{1 \mathrm{D}} \leq x_{\mathrm{D}} \leq x_{2 \mathrm{D}}\right), \\
\left.\frac{\partial p_{\mathrm{FD}}}{\partial x_{\mathrm{D}}}\right|_{x_{\mathrm{D}}=x_{1 \mathrm{D}}} & =\frac{\omega_{\mathrm{V}} R_{\mathrm{D}}^{3}}{L_{2 \mathrm{D}} W_{\mathrm{D}}} \frac{d p_{\mathrm{VD}}}{d t_{\mathrm{D}}}, \\
\left.\frac{\partial p_{\mathrm{FD}}}{\partial x_{\mathrm{D}}}\right|_{x_{\mathrm{D}}=x_{2 \mathrm{D}}} & =\frac{1}{L_{2 \mathrm{D}} W_{\mathrm{D}}}, \\
\left.p_{\mathrm{FD}}\right|_{x_{\mathrm{D}}=x_{1 \mathrm{D}}} & =p_{\mathrm{VD}}, \\
\left.p_{\mathrm{FD}}\right|_{x_{\mathrm{D}}=x_{2 \mathrm{D}}} & =p_{\mathrm{wD}}, \\
\left.p_{\mathrm{FD}}\right|_{t_{\mathrm{D}}=0} & =\left.p_{\mathrm{VD}}\right|_{t_{\mathrm{D}}=0}=\left.p_{\mathrm{wD}}\right|_{t_{\mathrm{D}}=0}=0 .
\end{aligned}
$$

In the above equations, dimensionless quantities are defined as follows:

$$
\begin{aligned}
x_{\mathrm{D}} & =\frac{x}{r_{\mathrm{w}}}, \\
x_{1,2 \mathrm{D}} & =\frac{x_{1,2}}{r_{\mathrm{w}}}, \\
p_{\mathrm{F}, \mathrm{V}, \mathrm{wD}} & =\frac{86.4 k_{\mathrm{F}} r_{w}}{q \mu B}\left(p_{i}-p_{\mathrm{F}, \mathrm{V}, \mathrm{w}}\right), \\
\omega_{\mathrm{F}, \mathrm{V}} & =\frac{\phi_{\mathrm{F}, \mathrm{V}} C_{\mathrm{tF}, \mathrm{tV}}}{\phi_{\mathrm{V}} C_{\mathrm{tV}}+\phi_{\mathrm{F}} C_{\mathrm{tF}}}, \\
t_{\mathrm{D}} & =\frac{3.6 k_{\mathrm{F}} t}{\mu\left(\phi_{\mathrm{V}} C_{\mathrm{tV}}+\phi_{\mathrm{F}} C_{\mathrm{tF}}\right) r_{w}^{2}}, \\
R_{\mathrm{D}} & =\left(\frac{\pi}{18}\right)^{1 / 3} \frac{R}{r_{\mathrm{w}}}, \\
L_{2 \mathrm{D}} & =\frac{L_{2}}{r_{\mathrm{w}}}, \\
W_{\mathrm{D}} & =\frac{W}{r_{\mathrm{W}}} .
\end{aligned}
$$

Employing the Laplace transform, the dimensionless Laplace solution can be obtained:

$$
\tilde{p}_{\mathrm{wD}}=\frac{a_{3} a_{1}+a_{3} a_{4} a_{2}}{a_{4} a_{2}-a_{1}}
$$

where $a_{1}=\sqrt{w_{\mathrm{F}} s}-\left(w_{\mathrm{V}} R_{\mathrm{D}}^{3} / L_{2 \mathrm{D}} W_{\mathrm{D}}\right) s, \quad a_{2}=\sqrt{w_{\mathrm{F}} s}+\left(w_{\mathrm{V}} R_{\mathrm{D}}^{3} /\right.$ $\left.L_{2 \mathrm{D}} W_{\mathrm{D}}\right) s, a_{3}=1 / L_{2 \mathrm{D}} W_{\mathrm{D}} s \sqrt{\omega_{\mathrm{F}} s}$, and $a_{4}=e^{-2 \sqrt{w_{\mathrm{F}}} s x_{1 \mathrm{D}}+2 \sqrt{w_{\mathrm{F}}} x_{2 \mathrm{D}}}$. 


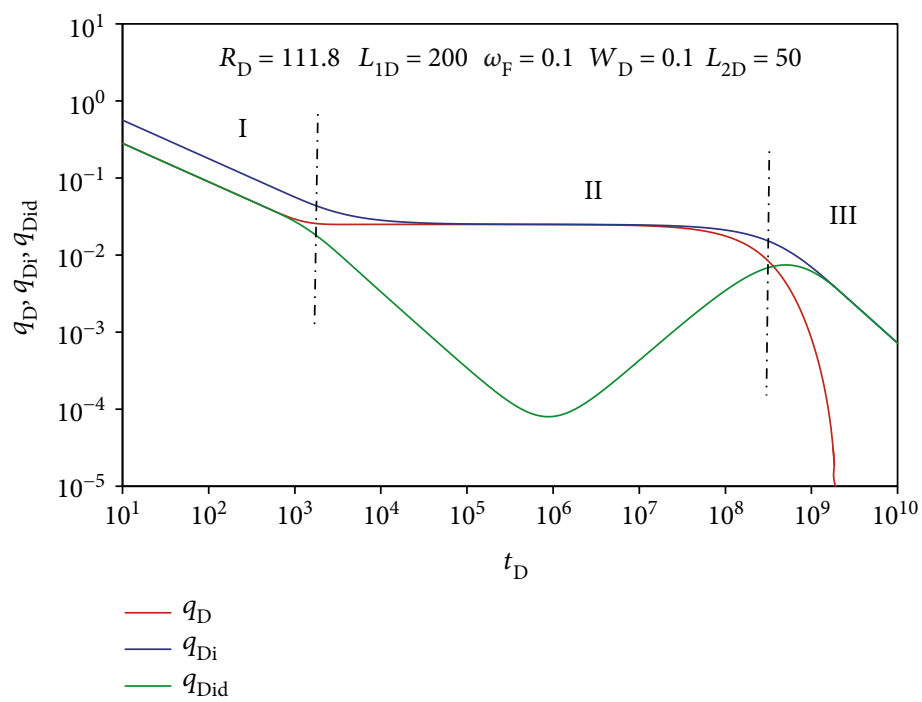

Figure 4: Vertical well rate transient analysis curve of single-cave and single-fracture model.

Using the Duhamel principle, the Laplace solution of the dimensionless bottomhole rate is

$$
\tilde{q}_{\mathrm{D}}(s)=\frac{1}{s^{2} \tilde{p}_{\mathrm{wD}}(s)} .
$$

2.1.3. Analysis of the Rate Transient Analysis Typical Curves and Influencing Factors. Numerical inversion is performed for the above-mentioned Laplace solutions. The logarithm of dimensionless production $q_{\mathrm{D}}$, dimensionless rate integral $q_{\text {Di }}$, and dimensionless rate integral derivative $q_{\text {Did }}$ are considered as the ordinate, and the logarithm of dimensionless time $t_{\mathrm{D}}$ is taken as the abscissa to draw the rate transient analysis curves of the oil well, as shown in Figure 4.

It can be seen from Figure 4 that the oil well rate transient analysis curves of the single-cave and single-fracture model can be divided into three stages. (I) The fracture linear flow stage, which is before the pressure wave reaches the cave, is mainly affected by the fracture's characteristic parameters. The dimensionless rate integral curve is parallel to the rate integral derivative curve, and the slope is $-1 / 2$. (II) The cave's response stage. This stage is mainly affected by the dissolved cave properties. The dimensionless integral curve tends to be horizontal, and the dimensionless integral derivative curve tends to "concave." The concave show the fluid supplementation from dissolved caves to fractures. (III) The boundary control flow stage. The dimensionless rate integral curve and the rate integral derivative curve overlap, showing a straight line with a slope of -1 .

The sensitivity analysis of the factors affecting the vertical well rate transient analysis curves with the single-cave and single-fracture model is as follows. Figure 5(a) shows the influence of dimensionless caves radius $R_{\mathrm{D}}$ on the rate transient analysis curves of the oil well. With the larger $R_{\mathrm{D}}$, the "concave" will be deeper and wider, and the cave's response stage will last longer. In addition, the boundary response stage will be later, i.e., the cave's storage capacity will be more significant, and the stable production capacity of the well will be better. Figure 5(b) shows the influence of fracture length $L_{1 \mathrm{D}}$ on the rate transient analysis curves of the oil well. $L_{1 \mathrm{D}}$ affects the duration of the fracture linear flow phase. With the longer $L_{1 \mathrm{D}}$, the parallel sections of the dimensionless rate integral and rate integral derivative curves are longer, the fracture linear flow stage lasts longer, and the cave response stage starts later. Figures 5(c) and 5(d) show the influence of fracture width $W_{\mathrm{D}}$ and fracture height $L_{2 \mathrm{D}}$ on the well rate transient analysis curve. $W_{\mathrm{D}}, L_{2 \mathrm{D}}$, and their products mainly affect fracture permeability and storage capacity. With a higher value, the fracture seepage performance is better, and the fluid will consume less energy. When the dimensionless curve in the fracture linear flow stage trends upward, the product of $W_{\mathrm{D}}$ and $L_{2 \mathrm{D}}$ becomes more significant, which weakens the influence of caves, and then the corresponding concave is relatively shallower and narrower. Figure 5(e) shows the influence of fracture storage capacity ratio $\omega_{\mathrm{f}}$ on the rate transient analysis curve. With the increase of $\omega_{\mathrm{F}}$, the fracture storage capacity will be better, and the rate transient analysis curve closer to upward. And as the corresponding $\omega_{\mathrm{V}}$ decreases, the concave becomes shallower and narrower.

2.1.4. Rate Transient Analysis Typical Curve of Simplified Model. If the fracture length $L_{1 \mathrm{D}}=0$, the single-cave and single-fracture model can be simplified to the single-cave model. In this case, the oil well is directly drilled in the large-scale cave, and the storage capacity and permeability of the surrounding matrix is negligible. The single-cave physical model is shown in Figure 6, and the single-cave seepage model is shown in Figure 7.

Figure 8 is the vertical well rate transient analysis curve of the single-cave model. We found that the oil well rate transient analysis curve of the single-cave model can be divided into two stages. (I) The cave response stage. In the cave response stage, the dimensionless rate integral curve and the rate integral derivative curve overlap and form a line with a slope of -1 . The wellbore is then directly drilled in the dissolved cave, which is equivalent to an enlarged wellbore 


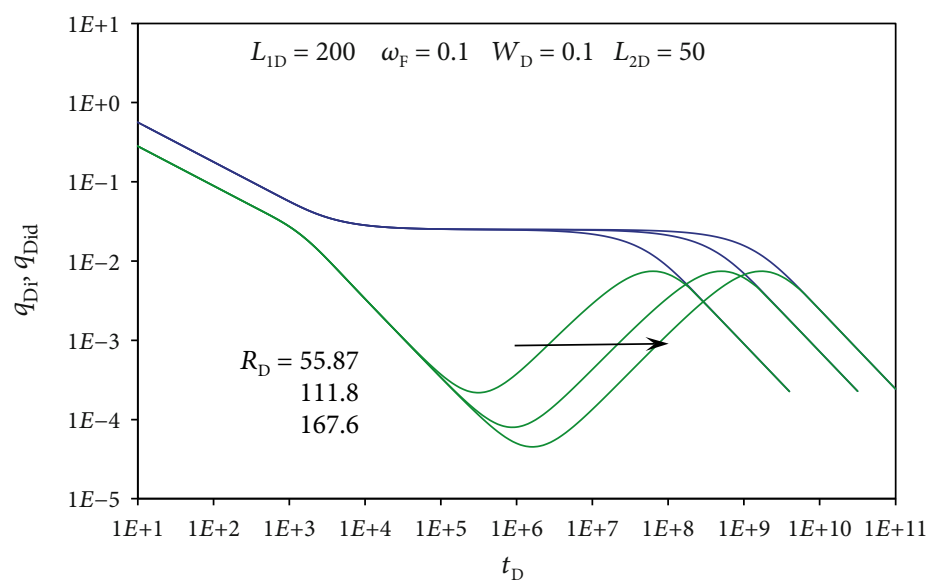

$$
-q_{\mathrm{Di}}
$$

(a)

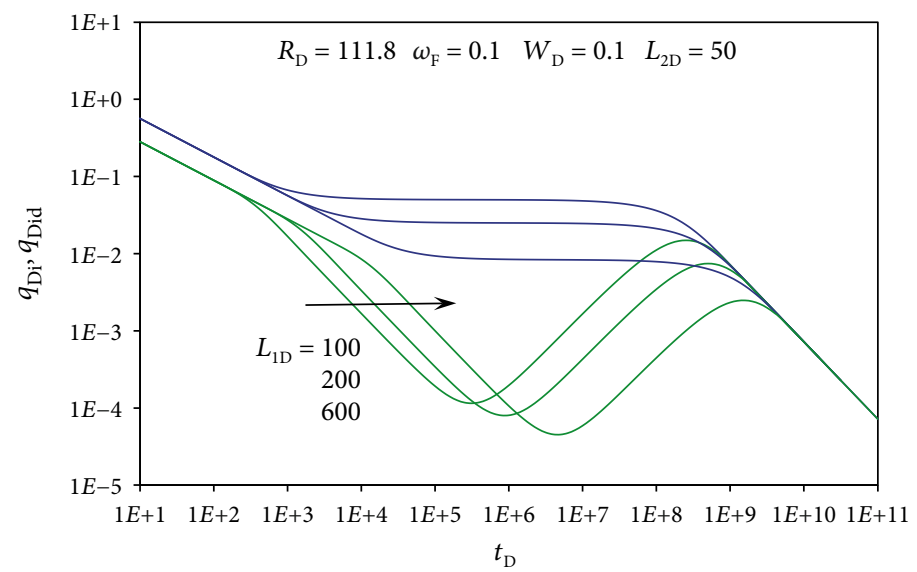

$$
\begin{aligned}
& q_{\mathrm{Di}} \\
& -q_{\mathrm{Did}}
\end{aligned}
$$

(b)

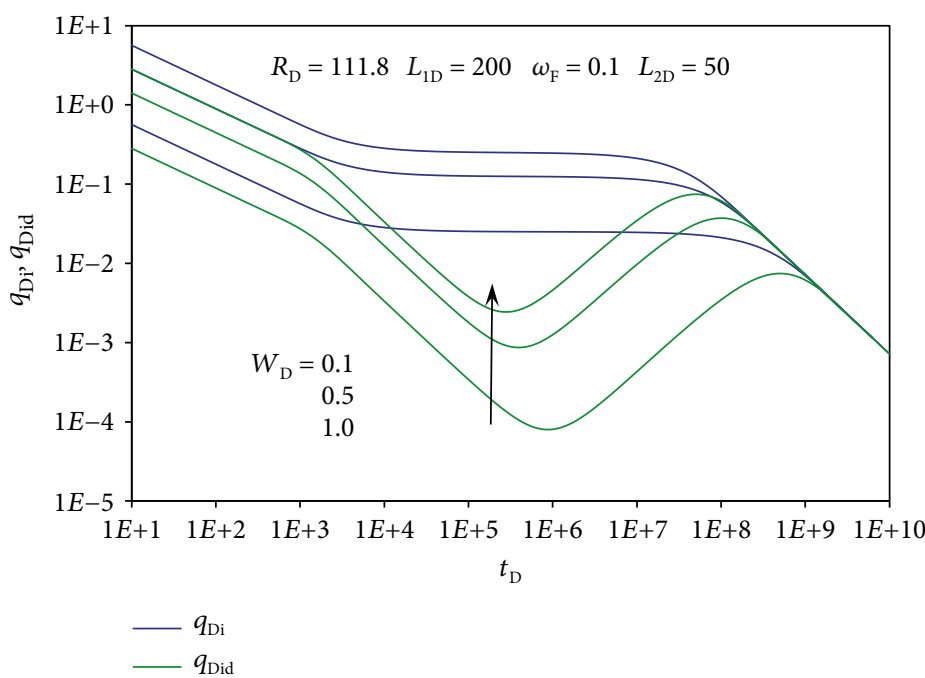

(c)

Figure 5: Continued. 

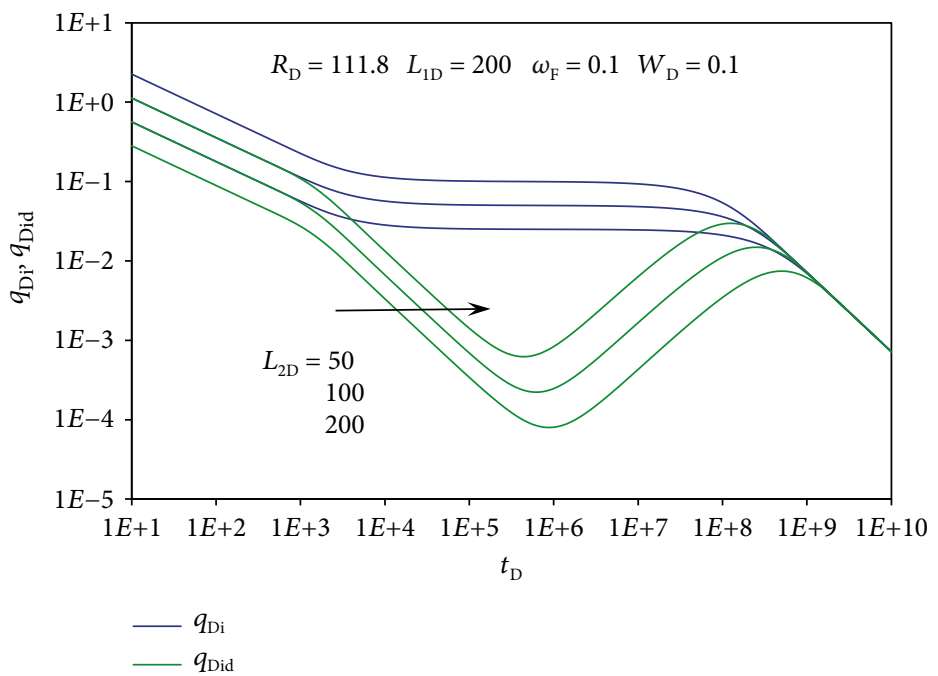

(d)

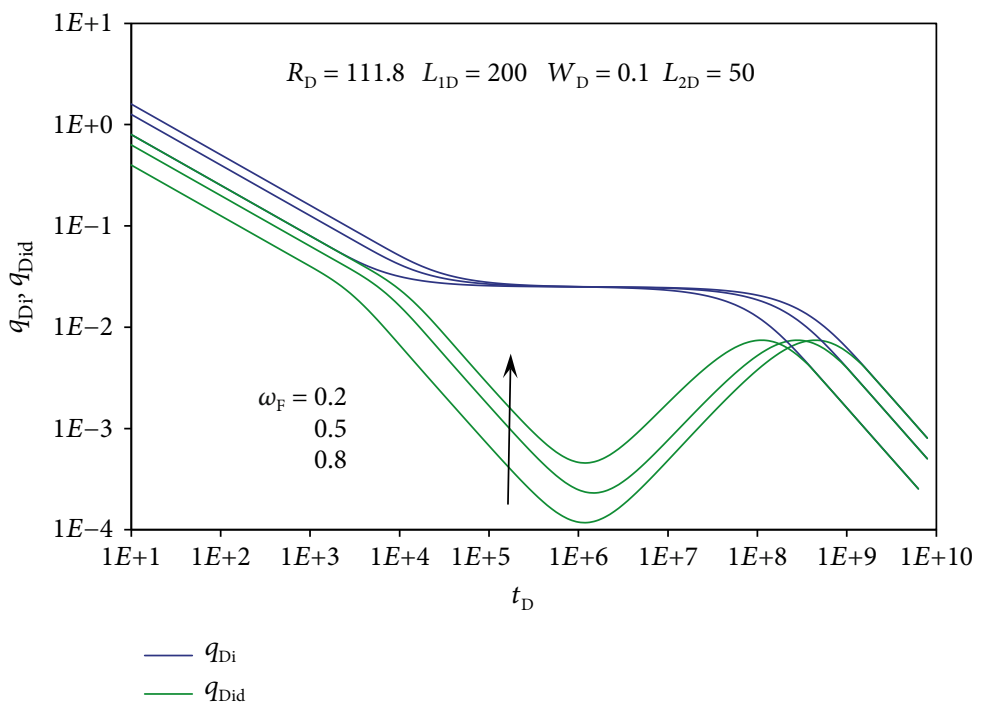

(e)

Figure 5: Vertical well rate transient analysis curves of single-cave and single-fracture model: (a) the influence of $R_{\mathrm{D}}$ on the rate transient analysis curve; (b) the influence of $L_{1 \mathrm{D}}$ on rate transient analysis curve; (c) the influence of $W_{\mathrm{D}}$ on rate transient analysis curve; (d) the influence of $L_{2 \mathrm{D}}$ on rate transient analysis curve; (e) the influence of $\omega_{\mathrm{F}}$ on rate transient analysis curve.

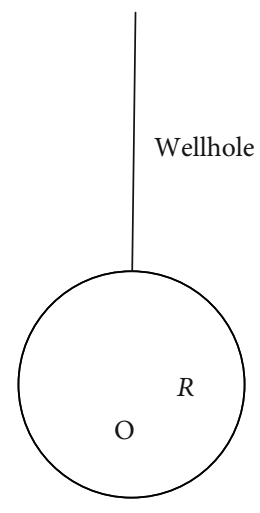

FIGURE 6: Single-cave physical model.

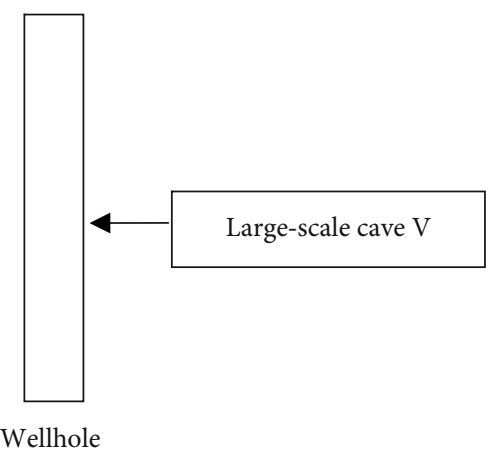

FIGURE 7: Single-cave seepage model. 


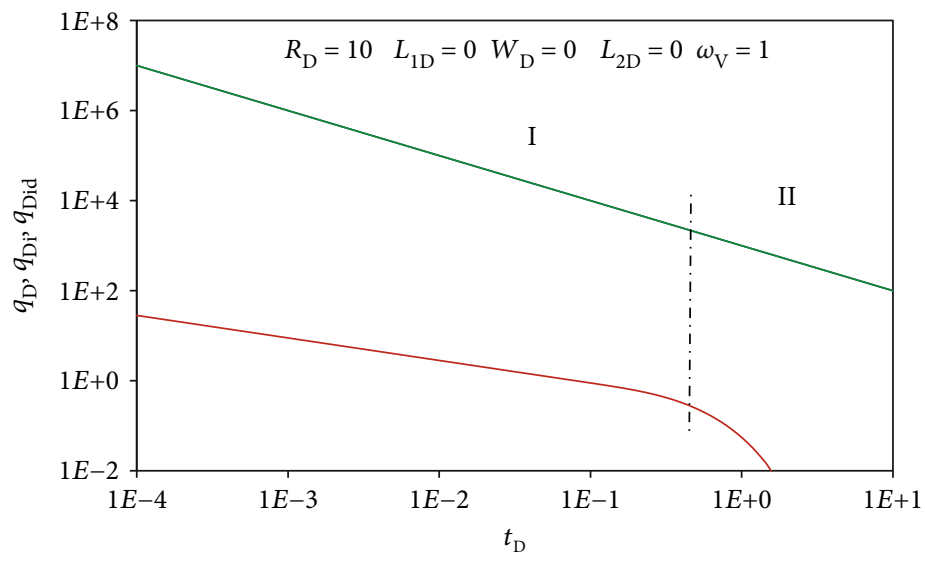

$$
-q_{\mathrm{D}}
$$

FIGURE 8: Vertical well rate transient analysis curve of single-cave model.

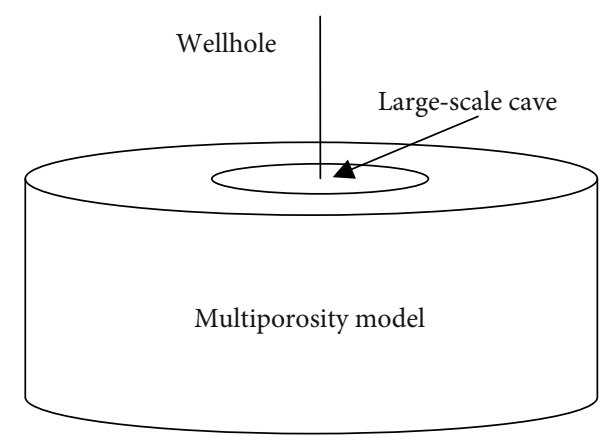

FIGURE 9: Single-cave and multimedium composite model.

and consistent with the wellbore storage effect. (II) The boundary control flow stage. For the free flow of large isolated caves, the pressure wave will quickly propagate to the boundary. The dimensionless rate integral curve coincides with the rate integral derivative curve and is also a straight line with a slope of -1 .

2.2. Single-Cave and Multiporosity Composite Model. When large-scale caves are developed in carbonate reservoirs and small-scale dissolved pores and fracture systems are evenly distributed around the caves, then the permeability and storage capacity of the matrix pores and fracture network around the caves must be taken into consideration. In this case, the simplified large-scale cave and multiple-porosity composite model can be used for reservoir characterization.

2.2.1. Physical Model. Figure 9 shows the composite physical model of single cave and multiple porosity. The oil well is directly drilled in large-scale dissolved caves. The area around the dissolved caves can be modeled as a single medium, double medium, or multiple medium model according to the characteristics of the small-scale pores, vugs and fractures. The fractured-caved reservoir is considered as a three-dimensional cylindrical composite model, in which the large-scale dissolved caves are internal cylinders. The cyl-

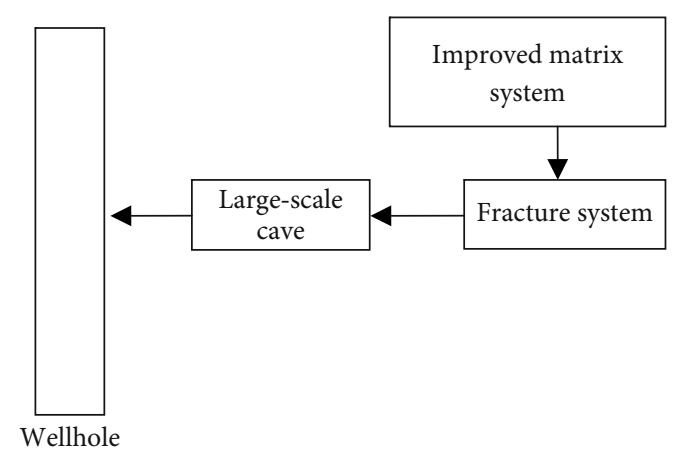

FIGURE 10: Single-cave and multimedium composite model.

inder height is $h$, the radius is $R_{\mathrm{V}}$, and the outside is multimedium and concentric with the cylinder. The fluid flow in large-scale dissolved cave is considered to be free flow, while that in the surrounded multiple-porosity model is considered to be seepage flow. Figure 10 is a fluid flow schematic. The surrounded improved matrix forms because of the development of small-scale caves.

\subsubsection{Establishment and Solution of the Mathematical Model.} The fluid flow capacity in large caves is extraordinarily strong and therefore considered to be free flow, i.e., the fluid flow in the caves produces truly little pressure drawdown. The dimensionless partial differential equation describing the composite model composed of large-scale caves and surrounding formation is as follows (considering improved matrix that develops small-scale caves):

$$
\begin{aligned}
\frac{\partial^{2} p_{\mathrm{fD}}}{\partial r_{\mathrm{D}}^{2}}+\frac{1}{r_{\mathrm{D}}}\left(\frac{\partial p_{\mathrm{fD}}}{\partial r_{\mathrm{D}}}\right)+\lambda_{\mathrm{im}}\left(p_{\mathrm{imD}}-p_{\mathrm{fD}}\right) & =\omega_{\mathrm{f}} \frac{\partial p_{\mathrm{fD}}}{\partial t_{\mathrm{D}}}, \\
\omega_{\mathrm{im}} \frac{\partial p_{\mathrm{imD}}}{\partial t_{\mathrm{D}}}+\lambda_{\mathrm{im}}\left(p_{\mathrm{imD}}-p_{\mathrm{fD}}\right) & =0 \\
1+\left.R_{\mathrm{D}} \frac{\partial p_{\mathrm{fD}}}{\partial r_{\mathrm{D}}}\right|_{r_{\mathrm{D}}=R_{D}} & =\left.R_{\mathrm{D}}^{2} \omega_{\mathrm{V}} \frac{\partial p_{\mathrm{fD}}}{\partial t_{\mathrm{D}}}\right|_{r_{\mathrm{D}}=R_{D}},
\end{aligned}
$$




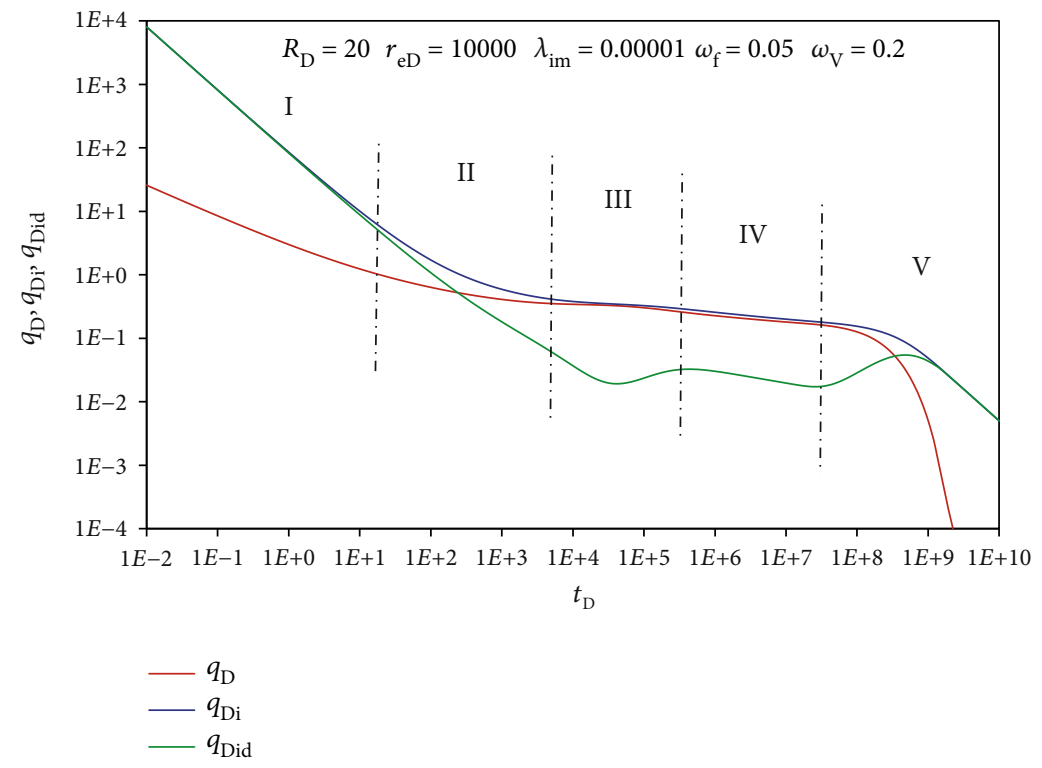

FIgURE 11: Oil well rate transient analysis curves of single-cave and multiple-porosity composite model.

$$
\begin{aligned}
\left.p_{\mathrm{fD}}\right|_{r_{\mathrm{D}}=R_{\mathrm{D}}} & =p_{\mathrm{wD}}=p_{\mathrm{vD}}, \\
\left.\frac{\partial p_{\mathrm{fD}}}{\partial r_{\mathrm{D}}}\right|_{r_{\mathrm{D}}=r_{\mathrm{eD}}} & =0, \\
\left.p_{\mathrm{imD}}\right|_{t=0} & =\left.p_{\mathrm{fD}}\right|_{t=0}=\left.p_{\mathrm{VD}}\right|_{t=0}=0 .
\end{aligned}
$$

In the above equations, dimensionless quantities are defined as follows:

$$
\begin{gathered}
p_{\mathrm{f}, \mathrm{im}, \mathrm{VD}}\left(r_{\mathrm{D}}, t_{\mathrm{D}}\right)=\frac{k_{\mathrm{f}} h}{1.842 \times 10^{-3} B q \mu}\left[p_{\mathrm{i}}-p_{\mathrm{f}, \mathrm{im}, \mathrm{V}}(r, t)\right], \\
r_{\mathrm{D}}=\frac{r}{r_{\mathrm{w}}}, r_{\mathrm{eD}}=\frac{r_{\mathrm{e}}}{r_{\mathrm{w}}}, R_{\mathrm{D}}=\frac{R}{r_{\mathrm{w}}}, \\
t_{\mathrm{D}}=\frac{\phi_{\mathrm{f}, \mathrm{im}, \mathrm{V}} C_{\mathrm{tf}, \mathrm{tim}, \mathrm{tV}}}{\left(\phi_{\mathrm{f}} C_{\mathrm{tf}}+\phi_{\mathrm{im}} C_{\mathrm{tim}}\right) \mu r_{\mathrm{w}}^{2}}, \omega_{\mathrm{f}, \mathrm{im}, \mathrm{V}}=\frac{\phi_{\mathrm{f}} C_{\mathrm{tf}}+\phi_{\mathrm{im}} C_{\mathrm{tim}}+\phi_{\mathrm{V}} C_{\mathrm{tV}}}{\phi_{\mathrm{f}}} \\
\lambda_{\mathrm{im}, \mathrm{v}}=\frac{\alpha_{\mathrm{im}} k_{\mathrm{im}}}{k_{\mathrm{f}}} r_{\mathrm{w}}^{2} .
\end{gathered}
$$

The dimensionless Laplace space solution expression can be written as Equation (7) by combining the above equations and the Laplace transformation.

$$
\tilde{p}_{\mathrm{wD}}=\frac{Y_{1} I_{0}\left(R_{\mathrm{D}} \sigma\right)+K_{0}\left(R_{\mathrm{D}} \sigma\right)}{s\left(Y_{3}-Y_{1} Y_{2}\right)},
$$

where

$$
\begin{aligned}
Y_{1} & =\frac{K_{1}\left(r_{\mathrm{eD}} \sigma\right)}{I_{1}\left(r_{\mathrm{eD}} \sigma\right)}, Y_{2}=R_{\mathrm{D}} \sigma I_{1}\left(R_{\mathrm{D}} \sigma\right)-R_{\mathrm{D}}^{2} \omega_{\mathrm{V}} s I_{0}\left(R_{\mathrm{D}} \sigma\right) \\
Y_{3} & =R_{\mathrm{D}} \sigma K_{1}\left(R_{\mathrm{D}} \sigma\right)+R_{\mathrm{D}}^{2} \omega_{\mathrm{V}} s K_{0}\left(R_{\mathrm{D}} \sigma\right) \\
\sigma & =\sqrt{s f(s)}=\sqrt{s\left(\omega_{f}+\frac{\lambda_{\mathrm{im}} \omega_{\mathrm{im}}}{\omega_{\mathrm{im}} s+\lambda_{\mathrm{im}}}\right)} .
\end{aligned}
$$

Using the Duhamel principle, the Laplace solution of the dimensionless bottomhole rate can be expressed as

$$
\tilde{q}_{\mathrm{D}}(s)=\frac{1}{s^{2} \tilde{p}_{\mathrm{wD}}(s)}
$$

2.2.3. Analysis of Rate Transient Analysis Typical Curves and the Influencing Factors. Similarly, we perform a numerical inversion on the Laplace solution above. The dimensionless oil well rate transient analysis curves form, as shown in Figure 11.

As shown in Figure 11, the characteristic curve of the composite model of a large-scale cave and multiple-porosity model can be divided into six stages. (I) The large-scale dissolved cave response stage is equivalent to an enlarged "wellbore," showing the wellbore storage effect. The dimensionless rate integral and rate integral derivative curves overlap into a straight line with a slope of -1: (II) the fractured peripheral multiple-porosity model linear flow and radial flow response stages, mainly related to the fracture properties of the multiple-porosity model; (III) the fluid flow from improved matrix to fractures in the surrounding multiple media, the 


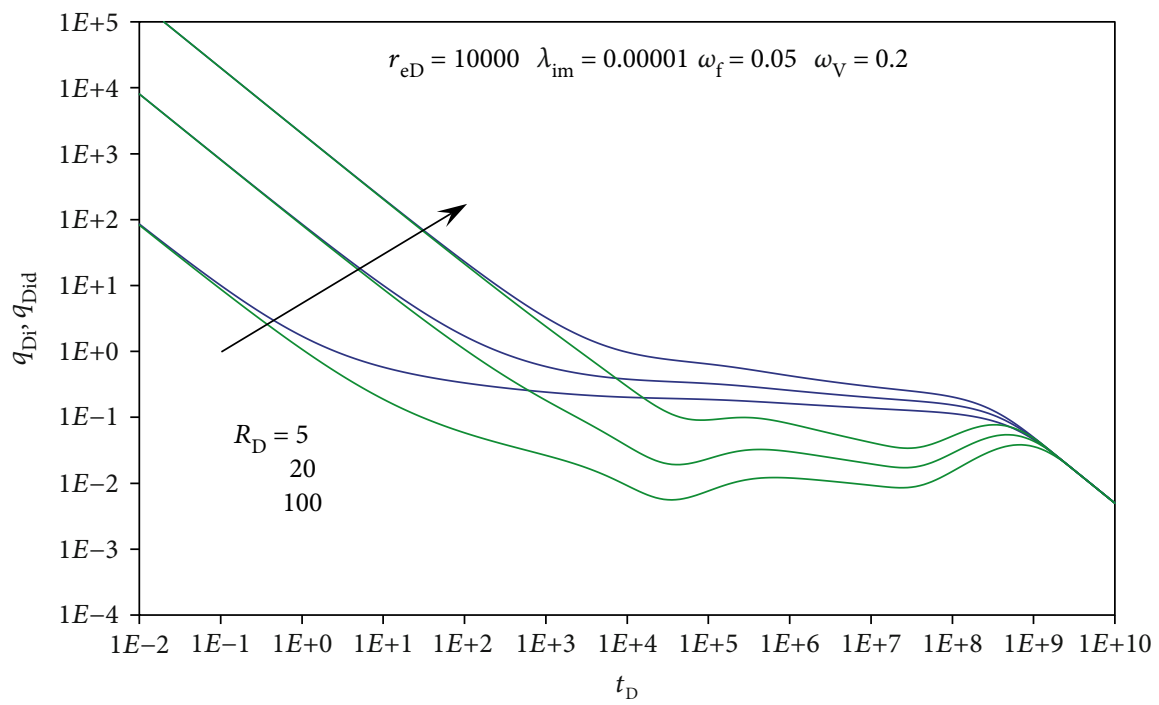

$$
\begin{aligned}
& -q_{\mathrm{Di}} \\
& -q_{\mathrm{Did}}
\end{aligned}
$$

(a)

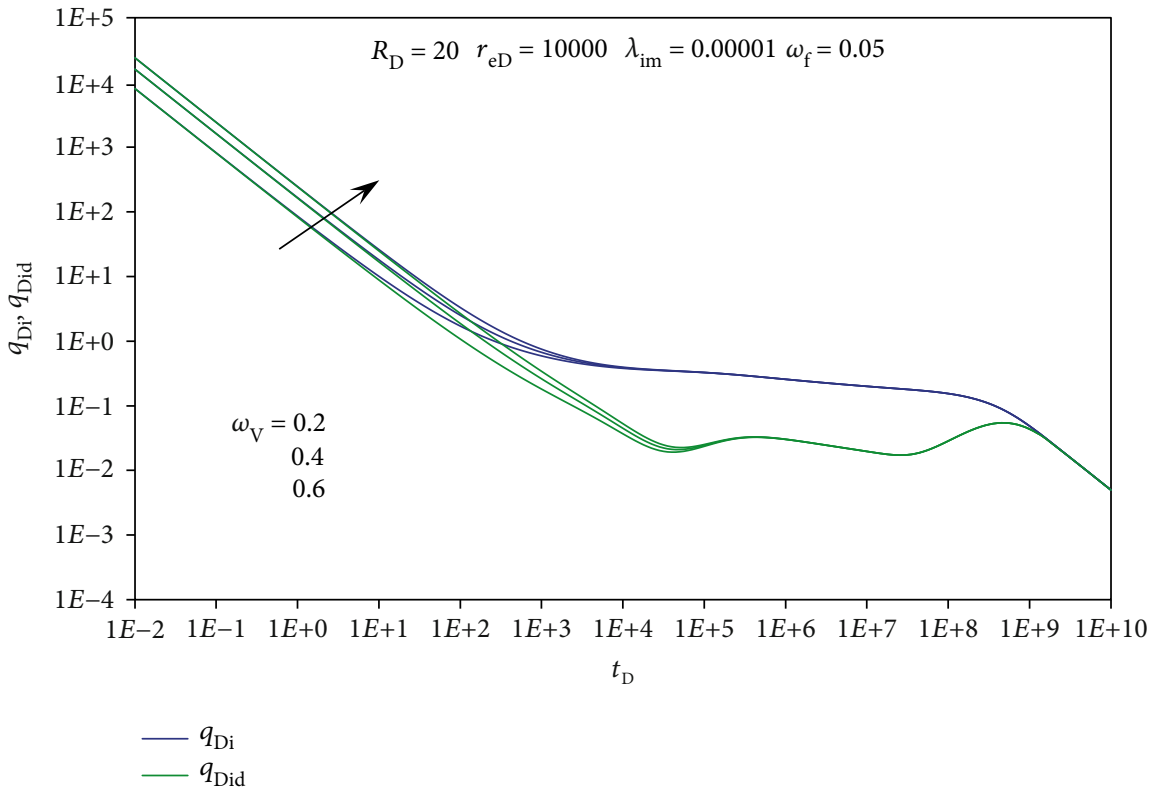

(b)

Figure 12: Continued. 


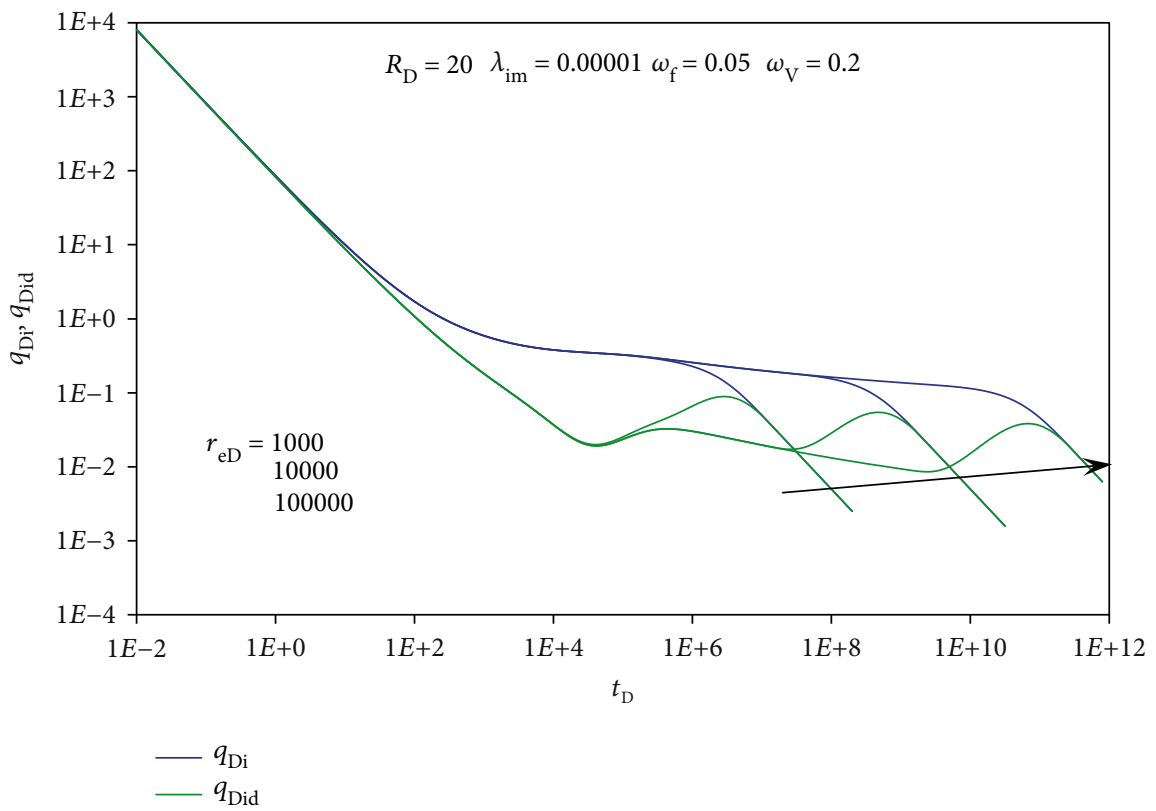

(c)

FIGURE 12: The influence of different parameters on the rate transient analysis curves: (a) the influence of $R_{\mathrm{D}}$ on the rate transient analysis curves; (b) the influence of $\omega_{\mathrm{V}}$ on the rate transient analysis curves; (c) the influence of $r_{\mathrm{eD}}$ on the rate transient analysis curves.

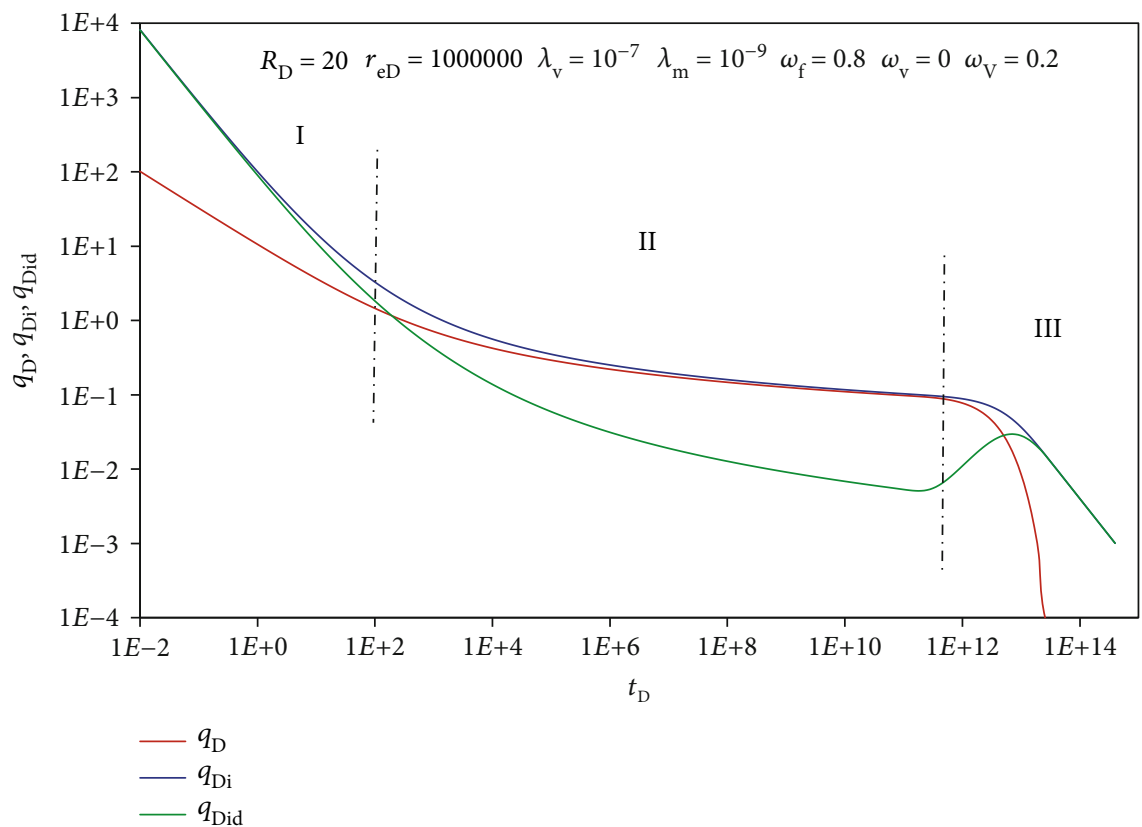

FIGURE 13: Oil well rate transient analysis curve with single-cave and single-medium composite model.

dimensionless rate integral derivative curve firstly appears "concave"; (IV) the radial flow stage of the model; and (V) the boundary control flow stage. The rate integral curve and the rate integral derivative curve overlap in a line with a slope of -1 for a closed boundary.

The sensitivity analysis of the influencing factors on the oil well rate transient analysis curve of the single-cave and dualporosity composite model is carried out as shown in Figure 12.

As shown in Figure 12(a), with the larger radius $R_{\mathrm{D}}$ of the large central cave, the intersecting line of the dimensionless rate integral and the rate integral derivative curve is close to upward. The stage's duration reflects the fact that the wellbore storage effect is longer. Figure 12(b) shows that the effect of $\omega_{\mathrm{V}}$ on the curve is similar to that of $R_{\mathrm{D}}$, but the effect of $\omega_{\mathrm{V}}$ is relatively small.

If $\omega_{\mathrm{V}}=0$ and $\omega_{\mathrm{f}}=0.8$, the single-cave and dual-porosity composite model can be simplified to a single-cave and single-medium composite model. Figure 13 shows the oil well rate transient analysis curves with a single cave and single medium composite model. 


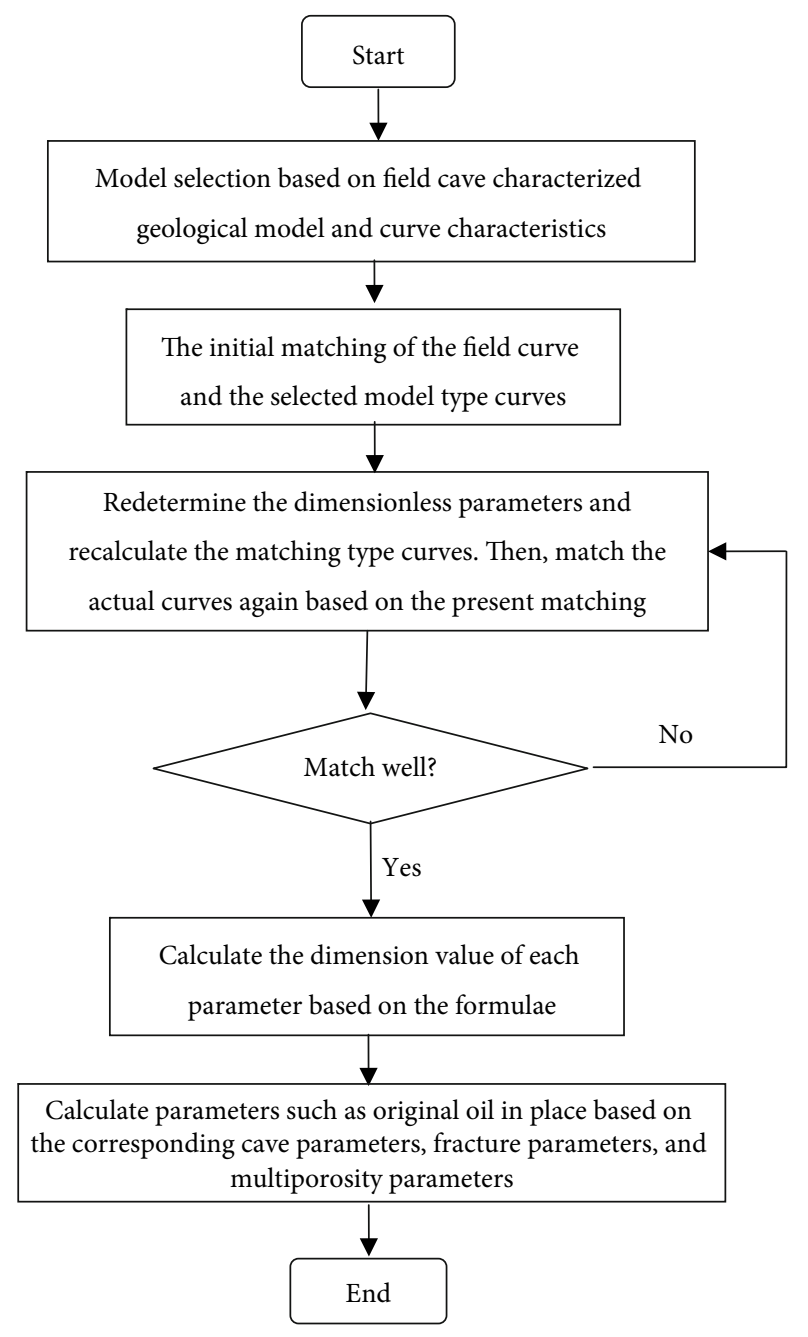

Figure 14: Flow chart of large-scale simplified model diagram matching.

\section{Application of Type Curves and the Evaluation Processes of Dynamic Parameters}

3.1. Dynamic Evaluation Method and Processes. For the above discretized simplified models of different fracturedcaved reservoirs developed in a large-scale single cave, the specific evaluation method and dynamic matching process of oil well and reservoir parameters are as follows:

(1) Obtain the oil well actual production data and calculate the normalized production rate, rate integral, and rate integral derivative curves $[22,23]$. The specific calculation method adopts Equations (10)-(13)

The equation for calculating the material balance pseudotime of the oil well:

$$
t_{\mathrm{d}}=\frac{N_{\mathrm{p}}}{q} .
$$

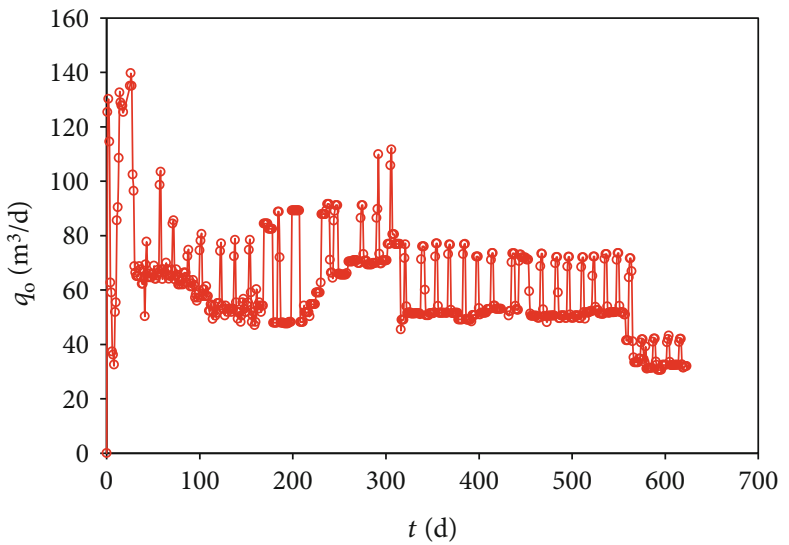

(a)

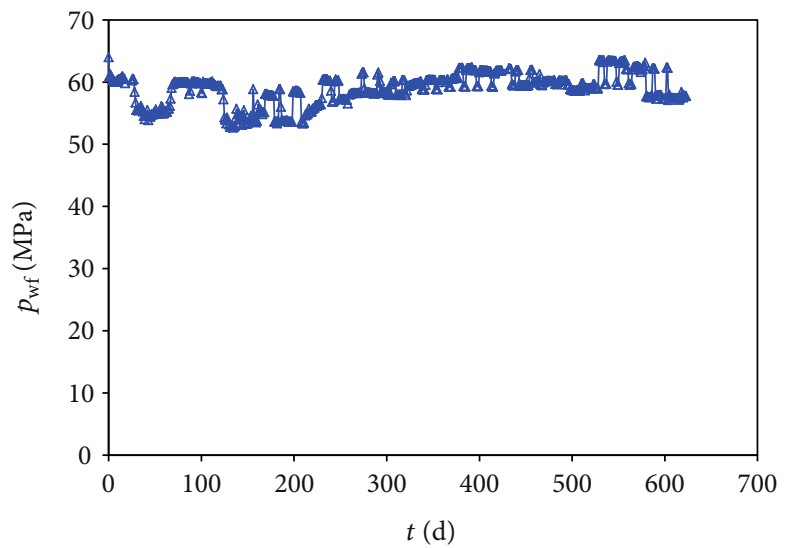

(b)

FIgURE 15: W well production dynamic curve: (a) daily oil production; (b) bottomhole pressure.

TABLe 1: Basic data of $\mathrm{W}$ oil well.

\begin{tabular}{lc}
\hline Initial formation pressure $(\mathrm{MPa})$ & 64 \\
Formation temperature $(\mathrm{K})$ & 424.45 \\
Effective formation thickness $(\mathrm{m})$ & 19 \\
Irreducible water saturation $(\%)$ & 20 \\
Porosity (\%) & 40 \\
Well radius (m) & 0.086 \\
Rock compressibility $\left(10^{-4} \mathrm{MPa}^{-1}\right)$ & 1.16 \\
Crude oil density $\left(\mathrm{kg} / \mathrm{m}^{3}\right)$ & 783.7 \\
Crude oil saturation $\mathrm{pressure}(\mathrm{MPa})$ & 14.6 \\
Crude oil viscosity $(\mathrm{mPa} \cdot \mathrm{s})$ & 1.5345 \\
Crude oil volume factor $\left(\mathrm{m}^{3} / \mathrm{m}^{3}\right)$ & 1.176 \\
Crude oil compression factor $\left(10^{-4} \mathrm{MPa}^{-1}\right)$ & 7.39 \\
\hline
\end{tabular}

The equation for calculating the normalized production rate of the oil well:

$$
q_{\mathrm{d}}=\frac{q}{\Delta p}=\frac{q}{p_{\mathrm{i}}-p_{\mathrm{wf}}} .
$$




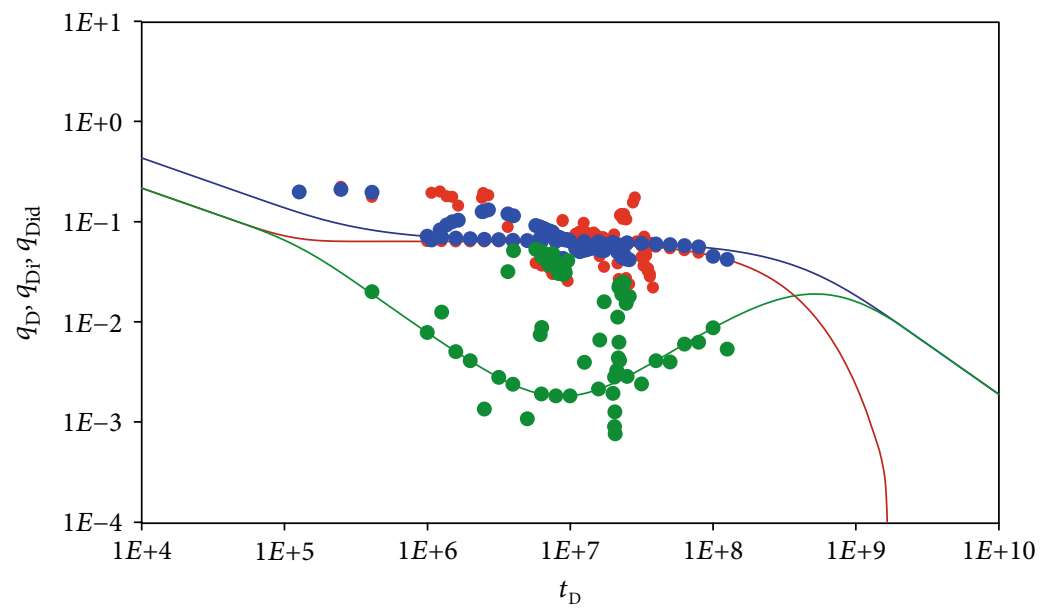

FIgURE 16: Matching results.

TABLE 2: Basic data of $\mathrm{W}$ oil well.

\begin{tabular}{lc}
\hline Fracture conductivity $(\mathrm{mD} \cdot \mathrm{cm})$ & 1427.3 \\
Cave radius $(\mathrm{m})$ & 90 \\
Oil well dynamic reserves $\left(10^{6} \mathrm{~m}^{3}\right)$ & 1.12 \\
Fracture storativity ratio & 0.2 \\
Dissolved cave storativity ratio & 0.8 \\
Fracture length $(\mathrm{m})$ & 100 \\
\hline
\end{tabular}

The equation for calculating the integral of normalized production rate for an oil well:

$$
q_{\mathrm{di}}=\frac{1}{t_{\mathrm{d}}} \int_{0}^{t_{\mathrm{d}}} q_{\mathrm{d}} \mathrm{d} \tau
$$

The equation for calculating the integral derivative of normalized oil well production rate:

$$
q_{\mathrm{did}}=t_{\mathrm{d}} \frac{\mathrm{d} q_{\mathrm{di}}}{\mathrm{d} t_{\mathrm{d}}}
$$

(2) In accordance with the initial static reservoir characterization model and the characteristics of the actual oil well production curve, a reasonable single-cave simplified model was initially selected

(3) In accordance with the initial static reservoir characterization model and the known dynamic and static description data, preliminary dimensionless parameters, such as large-scale dissolved caves and large fractures were assigned, and the dimensionless curves were initially matched with the field normalized curves

(4) We adjust the dimensionless parameters of largescale dissolved caves, large fractures, or multiple porosity according to the initial matching and the analysis of the influence of various factors on the curve. We also recalculate the dimensionless curves. In addition, the normalized production rate curve is moved to fit the well actual curve and to record the given dimensionless cave parameters, fracture parameters, or multiple-porosity model parameters. We obtain the actual reservoir parameters through the following specific equations

$$
\begin{aligned}
k_{\mathrm{F}} & =\frac{\mu B}{86.4 r_{w}}\left(\frac{q_{\mathrm{d}}}{q_{\mathrm{D}}}\right)_{M}, \\
r_{\mathrm{wa}} & =\sqrt{\frac{3.6 k_{\mathrm{F}}}{\mu\left(\phi_{\mathrm{F}} C_{\mathrm{tF}}+\phi_{\mathrm{V}} C_{\mathrm{tV}}\right)}\left(\frac{t_{\mathrm{d}}}{t_{\mathrm{D}}}\right)_{M},} \\
L_{F} & =L_{\mathrm{D}} r_{\mathrm{wa}}, \\
r_{\mathrm{e}} & =r_{\mathrm{eD}} r_{\mathrm{wa}}, \\
R & =\frac{R_{\mathrm{D}} r_{\mathrm{wa}}}{(\pi / 18)^{1 / 3}} .
\end{aligned}
$$

For the series model:

$$
N=\frac{4}{3} \pi R^{3} \phi_{\mathrm{V}}\left(1-S_{w c}\right)+L_{F} L_{F 2} W_{F} \phi_{F}\left(1-S_{w c}\right) .
$$

For the composite model:

$$
N=\frac{1}{C_{\mathrm{t}}}\left(\frac{t_{\mathrm{d}}}{t_{\mathrm{Dd}}}\right)\left(\frac{q_{\mathrm{d}}}{q_{\mathrm{Dd}}}\right)+\pi R^{2} h \phi_{\mathrm{V}}\left(1-S_{w c}\right) .
$$

Since we did not normalize the boundary control flow for the oil well rate transient analysis chart of the simplified discrete multiscale model (where seepage-free flow coupling is considered), we therefore have to comprehensively evaluate the initial static characterization model of the fracturedcaved reservoir (i.e., basic oil well-setting data, seismic data, well logging data, and other dynamic and static data) to 


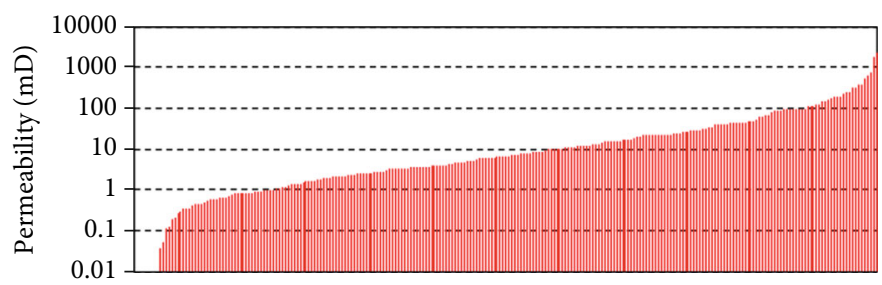

Figure 17: Histogram of single-well permeability evaluation results in Halahatang Oilfield.

obtain more accurate reservoir parameters and oil well parameters in the final fitting.

3.2. Programming of Dynamic Evaluation Method. We use $\mathrm{C} \#$ to compile a program to evaluate the corresponding reservoir parameters and oil well parameters in the discrete, simplified, and large-scale dissolved caved reservoir. The program includes four modules: crude oil and formation water physical parameter definition module, oil well production data input and analysis module, fitting model selection module, and specific matching module. The fitting model selection module can determine the number of large-scale fractures and caves and the contact relationship according to the known parameters. Then, the appropriate fitting model can be selected (Figure 14).

\section{The Application of Conventional Type Curves in Simplified Large-Scale Discrete Fracture-Cave Model}

We perform the dynamic evaluation of reservoir parameters using the $\mathrm{W}$ oil well of a fractured-caved reservoir in the Halahatang fractured-caved carbonate oilfield in Tarim Basin as a case study. Figure 15 shows the production curve of well $\mathrm{W}$ with high oil pressure, stable pressure, and stable production.

Based on the regular curve and static characterization model of well $\mathrm{W}$, we can conclude that the well is indirectly connected to large caves through fractures. It is located on the west slope of a low uplift to the south of the uplift in the north of Tarim Basin. The productive zone is Ordovician and lies at a vertical depth of $6579 \mathrm{~m}$. According to the current analysis, it can be simplified as a series model with a single cave and a single fracture, and this model can be used for fitting. Table 1 shows the basic data of the $\mathrm{W}$ well. The final matching results are shown in Figure 16, and the parameters obtained by matching are shown in Table 2 .

The dynamic controlled reserves of this well calculated by the material balance method are $1.14 \times 10^{6} \mathrm{~m}^{3}$, and the gap between this result and the result calculated by the method proposed in this article is relatively small. The type curve matching method can also accurately determine other caved parameters, large fracture parameters, and multiple surrounding porosity model parameters. If we consider the free flow of the cave, the result is more accurate. Through characterization of all wells in the Halahatang fractured-caved carbonate oilfield, we found that the single well dynamic reserves are mostly around $0.1 \times 10^{6} \mathrm{~m}^{3} \sim 1 \times 10^{6} \mathrm{~m}^{3}$, the permeability is mostly around $1 \mathrm{mD} 100 \mathrm{mD}$ (Figure 17), and

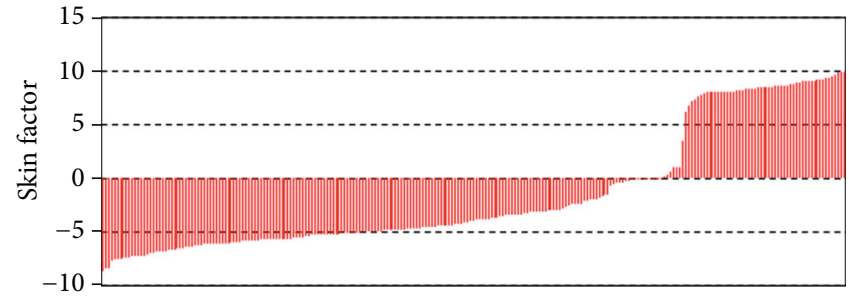

Figure 18: Distribution histogram of skin factor evaluation results of a single well in Halahatang Oilfield.

the skin factor is mostly less than 0 (Figure 18). An accurate understanding of the scale of reserves and of reservoir parameters can further guide reservoir development.

\section{Conclusion}

(1) For fractured-caved reservoirs with large-scale single dissolved cave, we propose simplified models for two large-cave models-a beaded model composed of caves and large fractures and a composite model composed of caves and surrounding reservoirs

(2) The large-scale discrete fractured-caved reservoir is simplified. According to the static fractured-caved characterization, the scale of fractures and caves and the contact connection relationship can be obtained. Then, the reservoir can be simplified into different single-cave combinations. Free flow is considered in large-scale dissolved caves, and seepage is considered in large fractures and surrounding reservoirs. The corresponding simplified model is established, and the mathematical model considering the coupling of seepage and free flow is solved analytically. The rate transient analysis typical curves of the oil well are obtained. A systematic theory and method are formed for the dynamic evaluation of reservoir parameters and oil well parameters

(3) By matching the oil well actual dynamic production data in a fractured-caved reservoir with the typical rate transient analysis type curves, parameters such as equivalent radius, fracture permeability, and the multiple-porosity model storage capacity ratio of each cave can be calculated. Finally, the dynamic controlled reserves of the different single-cave reservoirs can be determined. These results provide parameters for rational development of the fractured-caved reservoir 


\section{Nomenclature}

$x_{1,2}: \quad$ Distance to the coordinate origin $(\mathrm{m})$

$x$ : $\quad$ Distance from an arbitrary point to the coor-

dinate origin $(\mathrm{m})$

$R: \quad$ Karst cave radius

$L_{1}: \quad$ Fracture length $(\mathrm{m})$

$W: \quad$ Fracture width $(\mathrm{m})$

$L_{2}: \quad$ Fracture height $(\mathrm{m})$

$r_{\mathrm{w}}$ : Well radius $(\mathrm{m})$

$p_{F}: \quad \quad$ Formation pressure in the large scale fracture at a specific time $(\mathrm{MPa})$

$p_{V}$ : $\quad$ Formation pressure in karst cave at a specific time $(\mathrm{MPa})$

$\omega_{F}: \quad$ Elastic storativity ratio of the large scale fracture

$t$ : $\quad$ Production time (h)

$\omega_{V}: \quad$ Elastic storativity ratio of karst cave

$p_{\mathrm{w}}: \quad$ Bottomhole pressure $(\mathrm{MPa})$

$k_{F}$ : $\quad$ Large scale fracture permeability $\left(\mu \mathrm{m}^{2}\right)$

$q$ : Well production rate $\left(\mathrm{m}^{3} / \mathrm{d}\right)$

$B$ : $\quad$ Oil formation volume factor $\left(\mathrm{m}^{3} / \mathrm{m}^{3}\right)$

$\mu: \quad$ Oil viscosity (mPa.s)

$\phi_{F}: \quad$ Large scale fracture porosity (decimal)

$\phi_{V}: \quad$ Karst cave porosity (decimal)

$C_{t F}$ : Total compressibility coefficient of large scale fractures $\left(\mathrm{MPa}^{-1}\right)$

$C_{t V}$ : Total compressibility coefficient of karst caves $\left(\mathrm{MPa}^{-1}\right)$

$p_{f}: \quad$ Formation pressure in the surrounded fracture at a specific time $(\mathrm{MPa})$

$\lambda_{\mathrm{im}}$ : $\quad$ Interporosity flow coefficient from improved matrix to fractures (decimal)

$p_{\text {im }}$ : $\quad$ Formation pressure in the surrounded improved matrix at a specific time $(\mathrm{MPa})$

$\omega_{\mathrm{f}}$ : $\quad$ Elastic storability ratio of the surrounded fracture

$\omega_{\text {im }}$ : $\quad$ Elastic storability ratio of the surrounded improved matrix

$\begin{array}{ll}r_{\mathrm{e}}: & \text { Drainage radius }(\mathrm{m}) \\ k_{\mathrm{f}}: & \text { Fracture permeability }\left(\mu \mathrm{m}^{2}\right)\end{array}$

$h: \quad$ Thickness of the reservoir $(\mathrm{m})$

$p_{\mathrm{i}}$ : $\quad$ Initial reservoir pressure $(\mathrm{MPa})$

$\phi_{\mathrm{f}}: \quad$ Fracture porosity (decimal)

$C_{\mathrm{tf}}: \quad$ Total compressibility coefficient of fractures $\left(\mathrm{MPa}^{-1}\right)$

$\phi_{\text {im }}: \quad$ Improved matrix porosity (decimal)

$C_{\text {tim }}$ : $\quad$ Total compressibility coefficient of improved matrix $\left(\mathrm{MPa}^{-1}\right)$

$\alpha_{\mathrm{im}}: \quad$ Interflow shape factor (decimal)

$k_{\mathrm{im}}$ : $\quad$ Improved matrix permeability $\left(\mu \mathrm{m}^{2}\right)$

$s: \quad$ Laplace variable

$I_{0}, K_{0}, I_{1}, K_{1}$ : Bessel function

$q_{\mathrm{D}}$ : $\quad$ Normalized production rate $\left(\mathrm{m}^{3} / \mathrm{d} / \mathrm{MPa}\right)$

$q_{\mathrm{Di}}$ : $\quad$ Normalized production rate integral $\left(\mathrm{m}^{3} / \mathrm{d} / \mathrm{MPa}\right)$

$q_{\text {Did }}:$

Normalized production rate integral derivative $\left(\mathrm{m}^{3} / \mathrm{d} / \mathrm{MPa}\right)$

$t_{\mathrm{d}}$ : Material balance pseudotime (d) $p_{\text {wf }}: \quad$ Well field bottomhole pressure $(\mathrm{MPa})$

$N_{\mathrm{p}}$ : $\quad$ Well cumulative production $\left(\mathrm{m}^{3}\right)$

$r_{\text {wa }}$ : $\quad$ Efficient well radius $(\mathrm{m})$

$N$ : $\quad$ Well dynamic controlled reserves $\left(\mathrm{m}^{3}\right)$

$S_{\mathrm{wc}}$ : $\quad$ Irreducible water saturation (decimal).

Subscript

D: Dimensionless

V: Large scale caves

F: Large scale fractures

im: Improved matrix

f: Homogeneous fractures.

\section{Data Availability}

The raw data will be uploaded if required.

\section{Conflicts of Interest}

The authors declare that they have no conflicts of interest.

\section{Acknowledgments}

The authors appreciate the permission granted by the Research Institute of Petroleum Exploration \& Development (RIPED), PetroChina, to publish this paper. This research was funded by the National Natural Science Foundation of China (NSFC) (Grant No. 51874346).

\section{References}

[1] D. K. Han, "Status and challenges for oil and gas field development in China and directions for the development of corresponding technologies," Engineering and Science, vol. 12, no. 5, pp. 51-57, 2010.

[2] K. Zhang, "Strategic replacement situation and outlook of China oil-gas production area," Petroleum Exploration and Development, vol. 39, no. 5, pp. 513-523, 2012.

[3] P. Popov, G. Qin, L. Bi et al., "Multi-scale methods for modeling fluid flow through naturally fractured carbonate karst reservoirs," in SPE Annual Technical Conference and Exhibition, Society of Petroleum Engineers, pp. 11-14, Anaheim, California, USA, 2007.

[4] B. Vik, K. Djurhuus, K. Spildo, and A. Skauge, "Characterisation of Vuggy Carbonates," in SPE/EAGE Reservoir Characterization and Simulation Conference, Abu Dhabi, UAE, 2007.

[5] C. R. Wilson and P. A. Witherspoon, "Steady state flow in rigid networks of fractures," Water Resources Research, vol. 10, no. 2, pp. 328-335, 1974.

[6] S. Lee, C. Jensen, and M. Lough, "Efficient finite-difference model for flow in a reservoir with multiple length-scale fractures," SPE Journal, vol. 5, no. 3, pp. 268-275, 2000.

[7] D. Gläser, R. Helmig, B. Flemisch, and H. Class, "A discrete fracture model for two-phase flow in fractured porous media," Advances in Water Resources, vol. 110, pp. 335-348, 2017.

[8] B. Flemisch, I. Berre, W. Boon et al., "Benchmarks for singlephase flow in fractured porous media," Advances in Water Resources, vol. 111, pp. 239-258, 2018.

[9] I. Berre, F. Doster, and E. Keilegavlen, "Flow in fractured porous media: a review of conceptual models and 
discretization approaches," Transport in Porous Media, vol. 130, no. 1, pp. 215-236, 2019.

[10] D. G. Wang, J. J. Sun, Y. Li, and H. Peng, “An efficient hybrid model for nonlinear two-phase flow in fractured lowpermeability reservoir," Energies, vol. 12, no. 15, p. 2850, 2019.

[11] Z. Q. Huang, J. Yao, Y. J. Li, C. C. Wang, and X. R. Lü, "Permeability analysis of fractured vuggy porous media based on homogenization theory," Science China Technological Sciences, vol. 53, no. 3, pp. 839-847, 2010.

[12] Z. Q. Huang, J. Yao, Y. Y. Wang, and K. Tao, "Numerical study on two-phase flow through fractured porous media," Science China Technological Sciences, vol. 54, no. 9, pp. 2412-2420, 2011.

[13] Z. Q. Huang, J. Yao, and Y. Y. Wang, “An efficient numerical model for immiscible two-phase flow in fractured karst reservoirs," Communications in Computational Physics, vol. 13, no. 2, pp. 540-558, 2013.

[14] H. Liu and X. H. Wang, "Pressure response characteristics in large scale cavity type reservoir," Journal of Southwest Petroleum University (Science \& Technology Edition), vol. 34, no. 4, pp. 94-99, 2012.

[15] Z. J. Kang, Y. Di, Y. Y. Zhao, and L. Huang, "Flow characteristics of the cave fluids in fractured-vuggy oil reservoirs," Petroleum Geology \& Oilfield Development in Daqing, vol. 33, no. 3, pp. 82-85, 2014.

[16] Y. Z. Wan and Y. W. Liu, "Three dimensional discretefracture-cavity numerical well test model for fractured-cavity reservoir," Chinese Journal of Theoretical and Applied Mechanics, vol. 47, no. 6, pp. 1000-1008, 2015.

[17] J. Yao, Z. Huang, Y. Li, C. Wang, and X. Lv, "Discrete fracturevug network model for modeling fluid flow in fractured vuggy porous media," in International Oil and Gas Conference and Exhibition in China Society of Petroleum Engineers, pp. 8-10, Beijing, China, 2010.

[18] W. Djatmiko and V. Hansamuit, "Pressure buildup analysis in karstified carbonate reservoir," in SPE Asia Pacific Oil and Gas Conference and Exhibition, Society of Petroleum Engineers, pp. 18-20, Brisbane, Queensland, Australia, 2010.

[19] C. J. Tan, J. Y. Lv, and G. R. Li, “Correlativity between productivity features and reservoir type in carbonate oil reservoirs of Tahe oilfield," Petroleum Geology and Recovery, vol. 8, no. 3, pp. 43-45, 2001.

[20] D. Sun, J. G. Pan, X. S. Yong et al., "Formation mechanism of vertical "long string beads" in carbonate reservoir," Oil Geophysical Prospecting, vol. 45, Supplement 1, pp. 101-104, 2010.

[21] J. M. Urquiza, D. N'Dri, A. Garon, and M. C. Delfour, "Coupling Stokes and Darcy equations," Applied Numerical Mathematics, vol. 58, no. 5, pp. 525-538, 2008.

[22] T. A. Blasingame and W. J. Lee, "Variable-rate reservoir limits testing," in Permian Basin Oil and Gas Recovery Conference, Society of Petroleum Engineers, pp. 13-14, Midland, TX, 1986.

[23] T. A. Blasingame and W. J. Lee, "The variable-rate reservoir limits testing of gas wells," in SPE Gas Technology Symposium, Society of Petroleum Engineers, pp. 13-15, Dallas, Texas, 1988. 